\title{
La théorie de de Broglie- Bohm comme facon rationnelle de compléter la mécanique quantique
}

\section{Jean Bricmont}

Université catholique de Louvain, Belgique

\section{Résumé}

La mécanique quantique, telle qu'elle est habituellement formulée, se limite à prédire les « résultats de mesure » et ne dit rien sur le monde en dehors des laboratoires. La théorie de de Broglie-Bohm, par contre, donne une dynamique de la matière en mouvement, où les particules sont guidées par une onde, qui est solution de l'équation de Schrödinger. Cette théorie permet de retrouver les prédictions habituelles de la mécanique quantique et explique ce qui se passe lors de «mesures », qui sont en fait des interactions entre un système macroscopique et le système quantique "mesuré », interactions qui sont entièrement décrites par la théorie, sans faire de la " mesure » un deus ex machina, comme c'est le cas dans la présentation usuelle de la mécanique quantique. 


\section{Abstract}

\section{The de Broglie-Bohm theory as a rational completion of quantum mechanics}

Quantum mechanics, as it is ordinary formulated, limits itself to predict " results of measurements" and does not speak of the world outside of laboratories. On the other hand, the de Broglie-Bohm theory, gives a dynamics of matter in motion, where particles are guided by the wave that solves Schrödinger's equation. This theory recovers the usual predictions of quantum mechanics and explains what happens during "measurements", the latter being interactions between a macroscopic system and a measured quantum system, interactions that are entirely described by the theory, without turning measurements into deus ex machina, as is done in the usual presentations of quantum mechanics.

\section{Introduction}

La théorie quantique est sans doute la théorie scientifique la plus spectaculairement confirmée qui soit. Elle est à la base d'un grand nombre de technologies et n'a jamais été mise en défaut.

Néanmoins, ce qu'elle signifie comme théorie physique a été débattu depuis ses origines et l'est encore. Une façon simple de poser la question est de savoir ce que veut dire, pour un système physique, d'avoir ou d'être représenté par un état quantique?

C'est-à-dire par un vecteur $\Psi$ dans un espace de Hilbert !

La réponse standard (orthodoxe) est que, d'une part, cet état évolue selon l'équation de Schrödinger (voir (I3) plus loin) ou une équation similaire, tant que l'on n'effectue pas de mesure sur le système. Cette évolution est continue dans le temps, déterministe et linéaire.

Mais, d'autre part, si l'on mesure dans le système physique représenté par l'état $\Psi$, une quantité « observable » représentée par un opérateur $A$, ayant une base de vecteurs propres $\Psi_{n}$ avec valeurs propres $\lambda_{n}$, alors on obtient le résultat $\lambda_{k}$ avec probabilité $\left|c_{k}\right|^{2}$, où

$$
\Psi=\sum_{n} c_{n} \Psi_{n}
$$

est le développement de l'état $\Psi$ dans la base des $\Psi_{n}{ }^{1}$. Comme (I) est un développement dans une base orthonormale, on a nécessairement que $\sum_{n}\left|c_{n}\right|^{2}=1$, ce qui permet d'inter-
préter chaque $\left|c_{k}\right|^{2}$ comme une probabilité.

(I) Nous mettons de côté ici la question des spectres continus, qui peuvent être traités de façon conceptuellement similaire. 
Et, après la mesure de l'observable représentée par l'opérateur $A$, l'état « saute » ou « est réduit » à l'état $\Psi_{k}$.

Cette opération de réduction est discontinue dans le temps (l'état « saute »), aléatoire (on ne donne que certaines probabilités $\left|c_{k}\right|^{2}$ pour ces sauts), et non linéaire (le résultat $\Psi_{k}$ est indépendant des coefficients $c_{k}$ dans $\left.(I)\right)$.

Le problème est que le seul sens attribué à l'état quantique dépend entièrement des mesures ; si l'on demande ce que signifie physiquement un état attribué à un système hors du laboratoire, aucune réponse claire n'est donnée (en dehors justement de ce qui se passerait si l'on amenait ce système dans le laboratoire pour le « mesurer »).

Mais comment a fonctionné par exemple l'évolution naturelle ? Les processus biologiques reposent en principe sur la chimie qui elle-même est fondée sur la mécanique quantique. Comment décrire ce qui se passait avant l'apparition d'observateurs humains ?

Et que se passe-t-il dans l'univers en dehors des laboratoires ?

Est-ce que vraiment la physique n'a pas d'autre but que de décrire ce qui se passe dans les laboratoires ? Et si c'était le cas, pourquoi construire des laboratoires ? En principe, les expériences servent à tester nos théories sur le Monde, mais si celles-ci ne disent rien sur le Monde, à quoi servent les expériences ?

Et pourquoi a-t-on cette dualité dans l'évolution de l'état quantique : une évolution continue dans le temps, déterministe et linéaire en dehors des mesures et une évolution discontinue dans le temps, aléatoire, et non linéaire pendant celles-ci?

Parfois une réponse pragmatique est donnée à ces questions : puisque cela fonctionne, pourquoi s'en faire? Mais c'est justement parce que cela fonctionne si bien qu'il vaut la peine de se demander pourquoi cela fonctionne si bien. Si la théorie marchait une fois sur deux, pour ainsi dire, ou si elle n'était qu'un « modèle » physique parmi d'autres, dont le domaine d'application est limité mais connu, alors il n'y aurait effectivement aucune question à se poser.

Nous allons d'abord discuter deux réponses, dont l'une au moins est sans doute implicite dans l'esprit des physiciens qui pensent que la mécanique quantique ne pose pas de problème conceptuel.

Après avoir expliqué pourquoi aucune de ces réponses n'est satisfaisante, nous introduirons une description des systèmes physiques plus détaillée que celle donnée par l'état quantique et qui permet effectivement de résoudre les problèmes conceptuels de la mécanique quantique : la théorie de de Broglie-Bohm. 


\section{Deux « solutions ") qui n'en sont pas}

La première « solution » est de considérer qu'une analyse du processus de mesure, qui traiterait l'appareil de mesure de façon quantique, permettrait d'éviter cette opération de réduction, ou plutôt la ferait apparaître comme une conséquence de l'évolution temporelle de l'état du système 'total' constitué par le système mesuré et l'appareil de mesure. Après tout, l'appareil de mesure est nécessairement macroscopique et l'objet quantique mesuré microscopique ; est-ce que la différence d'échelle entre les deux pourrait nous permettre de résoudre le problème? On pourrait raisonner par analogie : les lois microscopiques sont réversibles sous le renversement du temps et les lois macroscopiques ne le sont pas, mais la mécanique statistique explique comment déduire les unes (les lois macroscopiques) des autres (les lois microscopiques) ${ }^{2}$. Est-ce que la réduction de l'état quantique ne pourrait pas être un phénomène du même type ?

Malheureusement, il n'en est rien.

\section{I Une analyse quantique de l'appareil de mesure}

Considérons un système très simple, composé du spin d'une particule (et dont nous négligerons la fonction d'onde, c'est-à-dire la partie spatiale de l'état quantique), dont l'état initial est :

$$
c_{1}|1 \uparrow\rangle+c_{2}|1 \downarrow\rangle
$$

où $|1 \uparrow\rangle$ dénote l'état de spin « up » dans une direction donnée, notée I (et $|1 \downarrow\rangle$ l'état « down ») et où $c_{1}, c_{2}$ sont des nombres (a priori, complexes). La formule (2) est un cas particulier de (I), et on a donc $\left|c_{1}\right|^{2}+\left|c_{2}\right|^{2}=1$.

L'appareil de mesure est représenté dans les figures I-3, et est réduit à un pointeur, qui est initialement dans une position horizontale et, à la fin de la mesure, pointe vers le haut ou vers le bas, selon que le spin mesuré est « up » ou « down ».

Pour décrire de façon quantique l'appareil de mesure, nous devons lui associer un état quantique. Soit $\varphi_{0}(z)$ la fonction d'onde initiale associée au pointeur, avec $\varphi_{0}(z)$ centrée en $z=0$, ce qui veut dire que le pointeur est comme dans la première image de la figure 1 . Évidemment, l'appareil de mesure est composé d'un grand nombre de particules et sa description complète ne peut être réduite à la variable $z$. Mais cette variable est une fonction des autres variables caractérisant le pointeur et est suffisante pour dire si le pointeur pointe vers le haut, vers le bas, ou horizontalement.

Soit :

$$
\Psi_{0}=\varphi_{0}(z)\left[c_{1}|1 \uparrow\rangle+c_{2}|1 \downarrow\rangle\right]
$$

(2) Voir par exemple [15]. 

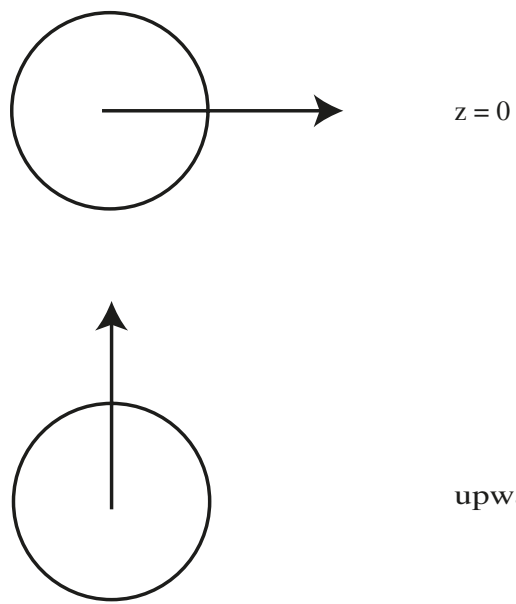

upward

Figure 1. Évolution du pointeur pendant une mesure lorsque l'état initial est donné par (4).

l'état qui décrit l'état initial du système composé du spin de la particule et du pointeur.

Pour comprendre ce qui se passe, considérons d'abord un autre état initial, qui correspond au spin de la particule étant dans l'état « up » :

$$
\Psi_{0}^{\uparrow}=\varphi_{0}(z)|1 \uparrow\rangle
$$

Alors, vu que le pointeur va aller vers le haut si le spin est « up », l'état final sera :

$$
\varphi^{\uparrow}(z)|1 \uparrow\rangle,
$$

où $\varphi^{\dagger}(z)$ correspond au pointeur étant comme dans la deuxième image de la figure $\mathrm{I}$.

De même, si nous partons de l'état initial :

$$
\Psi_{0}^{\downarrow}=\varphi_{0}(z)|1 \downarrow\rangle,
$$

l'état final sera : $\varphi^{\downarrow}(z)|1 \downarrow\rangle$, où $\varphi^{\downarrow}(z)$ correspond au pointeur étant comme dans la deuxième image de la figure 2 .

Voyons maintenant ce qui se passe si l'état initial est $\Psi_{0}$ (voir (3)). Vu que cet état est une combinaison linéaire de deux autres états, $\Psi_{0}^{\uparrow}$ (voir (4)) and $\Psi_{0}^{\downarrow}$ (voir (5)), et que l'évolution temporelle est linéaire, le résultat final sera nécessairement : 

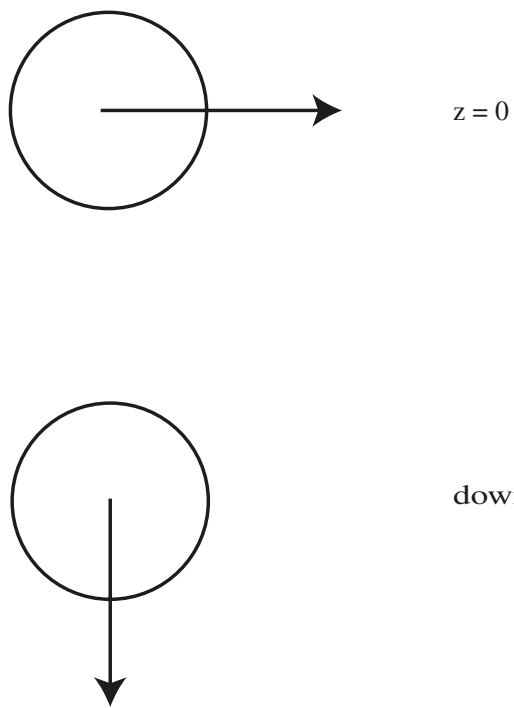

downward

Figure 2. Évolution du pointeur pendant une mesure lorsque l'état initial est donné par (5).

$$
c_{1} \varphi^{\uparrow}(z)|1 \uparrow\rangle+c_{2} \varphi^{\downarrow}(z)|1 \downarrow\rangle
$$

En ce qui concerne le pointeur, on ne peut interpréter cet état que comme une « superposition » de deux états macroscopiques différents : l'un où le pointeur est orienté vers le haut $\varphi^{\dagger}(z)$ et un autre où il est orienté vers le bas $\varphi^{\downarrow}(z)$. C'est illustré symboliquement dans la figure 3 .

Le problème qui se pose alors est que (6) ne représente nullement l'état de l'appareil de mesure tel que nous le connaissons. Le pointeur est orienté soit vers le haut, soit vers le bas, mais n'est pas dans une superposition des deux ! Ou, si l'on préfère, la description complète de l'appareil après la mesure n'est sûrement pas une superposition, puisqu'une description plus fine (vers le haut ou vers le bas) est obtenue simplement en regardant le résultat. Le problème est que les mesures ont des résultats bien définis, et le formalisme de la mécanique quantique ne rend pas compte de ce fait. II est parfois suggéré que le problème soulevé ici ne se pose pas parce qu'il est impossible de produire des effets d'interférence entre l'état du pointeur orienté vers le haut et celui du pointeur orienté vers le bas comparables à ce qu'on peut faire pour des systèmes composés d'une ou de peu de particules. Ceci est vrai (on appelle ce phénomène la décohérence), mais cela ne résout pas le problème, à savoir que la mécanique quantique ne rend pas compte de la réalité. Tout ce que cela prouve est qu'en pratique, on peut prendre l'état qu'on observe (pointeur orienté vers le haut ou pointeur orienté vers le bas) et oublier l'autre sans risquer de se 

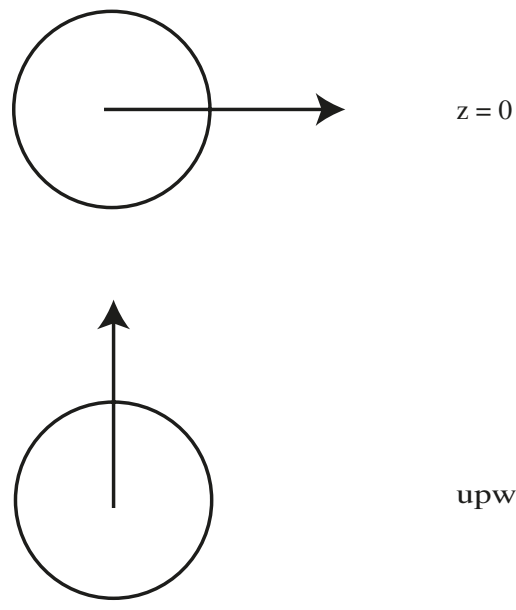

upward

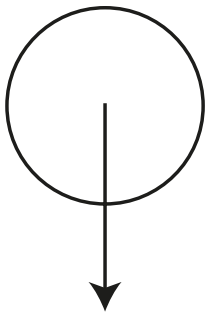

downward

Figure 3. Évolution du pointeur pendant une mesure lorsque l'état initial est donné par (3).

tromper en ce qui concerne l'évolution future du système. Mais le problème conceptuel, à savoir le rôle central de l'observateur, subsiste ${ }^{3}$.

Pour rendre les choses plus dramatiques, on peut coupler l'appareil à un chat, comme l'a suggéré Schrödinger [20] : on imagine un chat dans une boite fermée et un mécanisme qui lie la position du pointeur à un marteau qui brise une fiole de poison, si le pointeur est orienté vers le haut et ne la brise pas, s'il est orienté vers le bas. Si la fiole est cassée, le poison tue le chat (voir la figure 4). Raisonnant comme ci-dessus, l'état complet, y compris celui du chat, devient :

$c_{1} \varphi^{\uparrow}(z)|1 \uparrow\rangle \mid$ chat mort $\rangle+c_{2} \varphi \downarrow(z)|1 \downarrow\rangle \mid$ chat vivant $\rangle$

De nouveau, on obtient une superposition macroscopique donnée par la « somme » d'un chat vivant et d'un chat mort, expression qui ne correspond certainement pas à la réalité et à laquelle il est difficile d'attribuer même un sens. Pour dire les choses autrement, cet

(3) Nous verrons dans la section 3.2 que ce phénomène de décohérence est important pour comprendre la théorie de de Broglie-Bohm. 


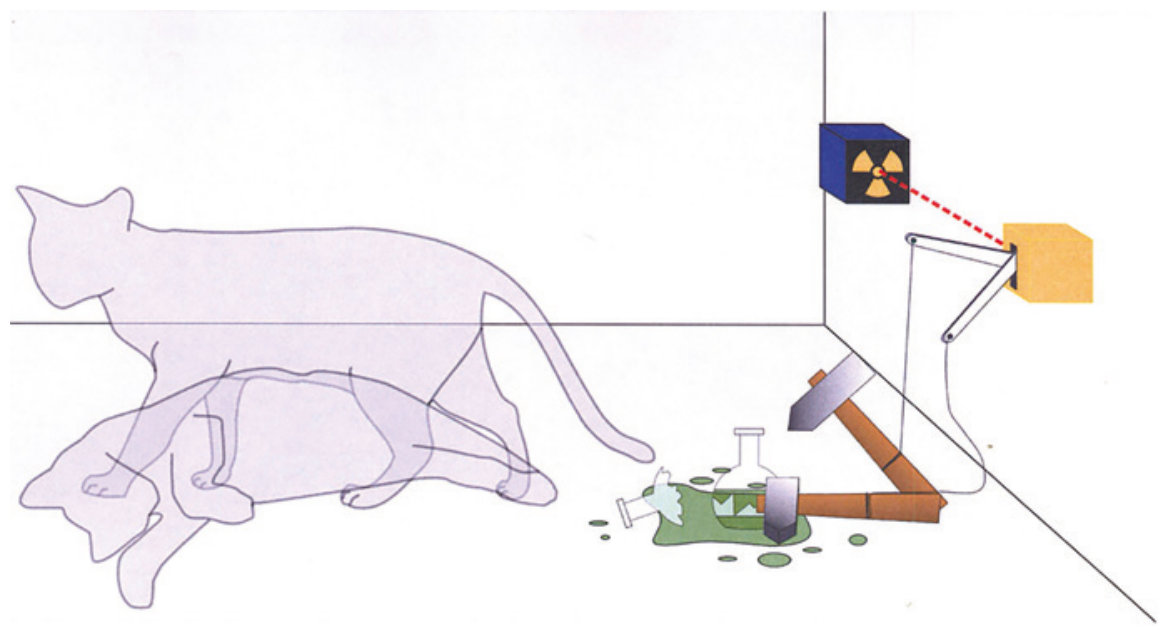

Figure 4. Le chat qui est à la fois vivant et mort.

Par Dhatfield (own work) [CC BY-SA 3.0] (http://creativecommons.Org/licenses/by-sa/3.0)], via Wikimedia Commons

argument montre que la mécanique quantique n'est manifestement pas complète, puisque, après la mesure, on peut décrire les systèmes physiques de façon plus complète que ne le fait l'état quantique.

Dire que l'on peut introduire une description physique plus complète que celle qui est donnée par l'état quantique revient, dans la terminologie usuelle sur les fondements de la mécanique quantique à introduire des « variables cachées ». Bien sûr, dans le cas du chat, le fait qu'il est vivant ou mort n'est nullement caché.

Mais on pourrait suggérer que des variables cachées existent à tous les niveaux : pas seulement pour le chat vivant ou mort, ou pour le pointeur dirigé vers le haut ou vers le bas, mais aussi pour la particule dont le spin est mesuré. On pourrait penser qu'un état tel que (2) signifie que le spin a une probabilité $\left|c_{1}\right|^{2}$ d'être « up » et une probabilité $\left|c_{2}\right|^{2}$ d'être « down » et que la mesure nous révèle simplement cette valeur du spin. Ou, plus précisément, on pourrait penser que, si l'on prépare un grand nombre de systèmes physiques caractérisés par l'état (2), une fraction égale à $\left|c_{1}\right|^{2}$ aura son spin « up » et une fraction égale à $\left|c_{2}\right|^{2}$ aura son spin « down ».

C'est ce qu'on appelle parfois l'interprétation statistique de l'état quantique. La règle de réduction de l'état quantique ne serait alors pas si mystérieuse, puisqu'une mesure nous apprendrait une propriété du système et, comme en probabilité classique, le fait d'apprendre quelque chose sur un système nous amène à modifier les probabilités que nous attribuons à ce système : par exemple si une pièce de monnaie est tombée sur une de ses faces, mais nous ne savons pas laquelle, nous attribuons une probabilité $(1 / 2,1 / 2)$ à chacune des faces ; mais si nous apprenons sur quelle face elle est tombée, nous changeons nos probabilités et nous attribuons une probabilité $I$ à la face sur laquelle elle est tombée et 0 à l'autre. 
De plus, cette façon de penser est très naturelle : si l'on dit que l'opération de mesure « mesure » quelque chose, alors on pense que ce qui est mesuré préexiste à la mesure : si je mesure la longueur d'une table, je suppose que la table a une certaine longueur avant que je ne la mesure ; sinon que voudrait dire le mot « mesure » ?

Cette vision des choses était, d'une certaine façon, celle d'Einstein lorsqu'il résuma sa position à propos de la mécanique quantique en 1949 :

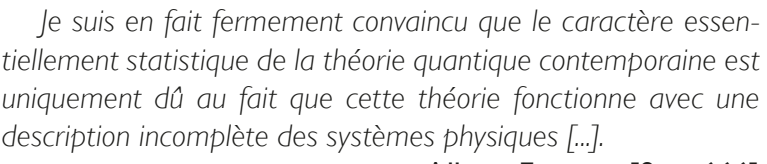

Albert Einstein [9, p. 666]

Si l'interprétation statistique de l'état quantique pouvait fonctionner nos problèmes seraient résolus. Malheureusement, des théorèmes mathématiques relativement peu connus mais faciles à démontrer montrent que cela ne fonctionne pas.

\subsection{L'impossibilité d'une interprétation statistique de l'état quantique}

Pour discuter de l'idée de variables cachées, il faut d'abord l'exprimer de façon précise. Soit $A$ une observable (nous ne distinguons pas ici entre l'observable physique, comme le spin, et l'opérateur qui la représente mathématiquement); dire que la mesure de $A$, dans un système physique particulier, va révéler la valeur que ce système a pour l'observable $A$ signifie qu'il existe une valeur $v(A)$ propre à ce système. Vu que différentes mesures peuvent être faites sur un système physique donné, il faut supposer qu'il existe une fonction $v$ définie sur un ensemble d'observables et qui donne, pour chaque observable, la valeur que cette observable a dans le système considéré.

Cette fonction doit être telle que $v(A)$ soit un des résultats possibles obtenus lors de la mesure de la quantité $A$, ce qui implique que la fonction $v(A)$ satisfasse deux propriétés :

$$
v(A) \in\{\text { valeurs propres de la matrice } \mathrm{A}\}
$$

et, $\forall A, B$, si $[A, B]=0$, alors

$$
v(A B)=v(A) v(B) .
$$

En effet, (7) dit simplement que si la mesure révèle une valeur préexistante de la quantité représentée par l'opérateur $A, v(A)$ doit être une des valeurs propres de cet opérateur (par exemple, pour le spin discuté ci-dessus, la valeur prise par $v$ sera « up » ou « down », ou 
+ | ou -I, si l'on veut associer à « up » et « down » des nombres). Quant à (8), elle exprime aussi une contrainte inévitable si la mesure en mécanique quantique révèle des valeurs préexistantes de quantités physiques. En effet, si les opérateurs $A$ et $B$ commutent alors, on peut les mesurer l'un et l'autre sans perturber les valeurs obtenues (du moins, d'après la mécanique quantique standard) ; on peut également mesurer la quantité $A B$ et les valeurs correspondantes doivent satisfaire (8).

Donc, les contraintes (7) et (8) peuvent être considérées comme purement empiriques. Elles ne dépendent pas de la véracité de l'ensemble de la théorie quantique, mais uniquement de certaines de ses prédictions qui sont amplement vérifiées expérimentalement.

Cependant, et là est le problème, on peut facilement démonter le

\section{Théorème sur l'inexistence de « variables cachées " $^{4}$}

II n'existe pas de fonction $v: \mathcal{A} \rightarrow \mathbb{R}$.

où $\mathcal{A}=$ ensemble des matrices sur un espace vectoriel de dimension au moins égale à 3 , telle que $\forall A, B \in \mathcal{A}$, les contraintes (7) et (8) soient satisfaites.

\section{Démonstration ${ }^{5}$}

Soient $\sigma_{x}^{i} \sigma_{y}^{i} i=1,2$ des paires de matrices de Pauli dans les directions $x$ et $y$ associées à deux « spins » différents, notés I et 2 , où l'usage de produits tensoriels est implicite :

$$
\sigma_{\alpha}{ }^{1}=\sigma_{\alpha} \otimes 1 \quad \sigma_{\alpha}^{2}=1 \otimes \sigma_{\alpha} \quad \alpha=x, y
$$

On a les relations :

$$
\begin{array}{cl}
\left(\sigma_{x}{ }^{i}\right)^{2}=\left(\sigma_{y}{ }^{i}\right)=1 & i=1,2 \\
\sigma_{x}{ }^{i} \sigma_{y}{ }^{i}=-\sigma_{y}{ }^{i} \sigma_{x}{ }^{i} & i=1,2 \\
\sigma_{\alpha}{ }^{1} \sigma_{\beta}{ }^{2}=\sigma_{\alpha}{ }^{2} \sigma_{\beta}{ }^{1} & \alpha, \beta=x, y .
\end{array}
$$

En utilisant les relations ci-dessus, on obtient :

$$
\begin{aligned}
& \sigma_{x}{ }^{1} \sigma_{y}{ }^{2} \sigma_{y}{ }^{1} \sigma_{x}{ }^{2} \sigma_{x}{ }^{1} \sigma_{x}{ }^{2} \sigma_{y}{ }^{1} \sigma_{y}{ }^{2}= \\
&-\sigma_{y}{ }^{2} \sigma_{y}{ }^{1} \sigma_{x}{ }^{2} \sigma_{x}{ }^{1} \sigma_{x}{ }^{1} \sigma_{x}{ }^{2} \sigma_{y}{ }^{1} \sigma_{y}{ }^{2}=-1 . \\
& X=\sigma_{x}{ }^{1} \sigma_{y}{ }^{2} \sigma_{y}{ }^{1} \sigma_{x}{ }^{2} \\
& \text { Introduisons les matrices : } \begin{aligned}
X & =\sigma_{x}{ }^{1} \sigma_{x}{ }^{2} \sigma_{y}{ }^{1} \sigma_{y}{ }^{2} .
\end{aligned}
\end{aligned}
$$

(4) Ce théorème est dû, sous différentes formes, à Bell [I], Kochen-Specker [II], Mermin [I6], Perez [17]. Voir [6], section 2.5 pour plus de détails.

(5) La démonstration donnée ici n'est valide que dans un espace vectoriel de dimension égale à 4 et peut facilement être étendue à un espace dont la dimension est un multiple de 4. Voir [6], Appendice 2.F pour plus de détails sur la situation générale. 
On a $\sigma_{x}{ }^{1} \sigma_{y}{ }^{2} \sigma_{y}{ }^{1} \sigma_{x}{ }^{2} \sigma_{x}{ }^{1} \sigma_{x}{ }^{2} \sigma_{y}{ }^{1} \sigma_{y}{ }^{2}=X Y$

et, par les relation de commutation et d'anti-commutation ci-dessus : $[X, Y]=0$

et donc $v\left(\sigma_{x}{ }^{1} \sigma_{y}{ }^{2} \sigma_{y}{ }^{1} \sigma_{x}{ }^{2} \sigma_{x}{ }^{1} \sigma_{x}{ }^{2} \sigma_{y}{ }^{1} \sigma_{y}{ }^{2}\right)=-1=v(X Y)=v(X) v(Y)$,

par les hypothèses (7) (qui implique $v(-1)=-1$ ) et (8).

On peut écrire $: X=A \cdot B$

Où $A=\sigma_{x}^{1} \sigma_{y}^{2} \quad B=\sigma_{y}^{1} \sigma_{x}^{2}$ et

$Y=C \cdot D$ où

$C=\sigma_{x}^{1} \sigma_{x}^{2} \quad D=\sigma_{y}^{1} \sigma_{y}^{2}$.

On peut vérifier que : $[A, B]=[C, D]=0$.

Et donc, en utilisant (8): $v(X)=v(A) v(B)$

Par conséquent :

$v(Y)=v(C) v(D)$

$-1=v\left(\sigma_{x}{ }^{1} \sigma_{y}{ }^{2} \sigma_{y}{ }^{1} \sigma_{x}{ }^{2} \sigma_{x}{ }^{1} \sigma_{x}{ }^{2} \sigma_{y}{ }^{1} \sigma_{y}{ }^{2}\right)=v(X Y)=v(X) v(Y)=v(A) v(B) v(C) v(D)$.

$A, B, C, D$ sont des produits de matrices qui commutent (parce qu'ayant des indices I et 2 ). Donc, toujours par (8) :

$$
\begin{aligned}
v(A) & =v\left(\sigma_{x}{ }^{1}\right) v\left(\sigma_{y}{ }^{2}\right) \\
v(B) & =v\left(\sigma_{y}{ }^{1}\right) v\left(\sigma_{x}{ }^{2}\right) \\
v(C) & =v\left(\sigma_{y}{ }^{1}\right) v\left(\sigma_{x}{ }^{2}\right) \\
v(D) & =v\left(\sigma_{y}{ }^{1}\right) v\left(\sigma_{y}{ }^{2}\right) .
\end{aligned}
$$

Ce qui implique :

$-1=v(A) v(B) v(C) v(D)$

$=v\left(\sigma_{x}^{1}\right) v\left(\sigma_{y}^{2}\right) v\left(\sigma_{y}{ }^{1}\right) v\left(\sigma_{x}^{2}\right) v\left(\sigma_{x}^{1}\right) v\left(\sigma_{x}^{2}\right) v\left(\sigma_{y}^{1}\right) v\left(\sigma_{y}^{2}\right)$

$=v\left(\sigma_{x}^{1}\right)^{2} v\left(\sigma_{y}^{2}\right)^{2} v\left(\sigma_{y}^{1}\right)^{2} v\left(\sigma_{x}^{2}\right)^{2}$. 
L'expression $v\left(\sigma_{x}^{1}\right)^{2} v\left(\sigma_{y}^{2}\right)^{2} v\left(\sigma_{y}^{1}\right)^{2} v\left(\sigma_{x}^{2}\right)^{2}$ est un nombre positif (en fait égal à $+\mathrm{I}$ ) et ne peut pas être égal à -I. Cette dernière relation est donc une contradiction et cela établit la non-existence d'une quelconque fonction $v$ ayant les propriétés supposées (7) et (8).

Soulignons que ce théorème ne signifie pas simplement qu'il n'existe pas d'états quantiques donnant une valeur bien déterminée simultanément à une série d'observables (ce qui est bien connu) mais quelque chose de bien plus profond : il est impossible d'ajouter des variables supplémentaires qui permettraient de comprendre l'opération de mesure comme signifiant ce que ce mot veut dire, c'est-à-dire, la mise en évidence d'une propriété préexistante à la mesure.

Une autre façon d'exprimer le contenu de ce théorème est de dire que l'appareil de mesure joue nécessairement un rôle « actif' en ce sens qu'il n'enregistre pas passivement une donnée déjà là (la valeur $v(A))$. C'est un aspect des choses sur lequel Bohr a toujours insisté, par exemple dans sa discussion avec Einstein en 1949, lorsqu'il soulignait :

[...] l'impossibilité de toute séparation nette entre le comportement des objets atomiques et leur interaction avec les instruments de mesure serrant à définir les conditions sous lesquelles le phénomène se manifeste.

Niels Bohr [5, p. 207] (italiques dans l'original)

Mais la mécanique quantique ordinaire ne nous dit absolument pas en quoi consiste ce rôle actif, puisque l'appareil de mesure est, dans le formalisme usuel, un deus ex machina.

\subsection{Conclusions}

Nous faisons face à un sérieux problème. Si nous essayons de comprendre la réduction de l'état quantique comme une propriété « émergente » au niveau macroscopique, nous voyons que le formalisme quantique n'en rend pas compte. Et si nous essayons de donner une interprétation statistique naturelle à l'état quantique et à sa mesure, nous arrivons à une contradiction.

Néanmoins, il est probable que de nombreux physiciens qui ne se posent pas de questions sur la mécanique quantique adhèrent implicitement à l'une de ces deux positions.

Il y a trois réactions possibles à cette situation :

I. Déclarer que le monde microscopique est incompréhensible et que nous ne pouvons parler que de ses manifestations expérimentales. Par exemple, que si l'on refait de nombreuses expériences avec un état tel que (2), dans une fraction $\left|c_{1}\right|^{2}$ des cas, à la fin de l'expérience, le pointeur des figures $(I, 2)$ sera dirigé vers le haut et dans une fraction $\left|c_{2}\right|^{2}$ des cas, il sera dirigé vers le bas.

2. Introduire, par fiat, une distinction nette entre monde quantique et monde classique. On décide que, par définition, certains objets comme les pointeurs ou les 
chats ont des propriétés bien définies, alors que les objets microscopiques n'en ont pas. Le problème avec cette approche est qu'elle introduit une coupure totalement arbitraire : si un atome est régi par les lois quantiques, que deux atomes le sont quid de dix atomes ou d'un million d'atomes ? Où mettre la séparation ? Soulignons que ce n'est pas la même chose qu'une limite (comme en mathématique ou lorsqu'on parle de "limite classique ») dont on peut s'approcher de plus en plus, parce qu'ici il s'agit d'un saut qualitatif entre « ne pas avoir de propriétés bien définies » et « en avoir ».

3. Finalement, on pourrait chercher à obtenir une théorie plus complète que la mécanique quantique ordinaire. Les théorèmes sur l'inexistence des variables cachées montrent que ce n'est pas si simple à trouver.

Les deux premières réactions sont associées à l'interprétation de Copenhague et sont essentiellement les positions « orthodoxes ». La troisième réaction sera discutée dans la section suivante et est illustrée par la théorie de de Broglie-Bohm.

\section{La théorie de de Broglie-Bohm}

La théorie de de Broglie-Bohm, élaborée par de Broglie en 1924-1927 (voir [7]) et redécouverte et développée par Bohm en 1952 [4] est une :

- théorie de « variables cachées »;

- qui ne sont nullement cachées ;

- théorie qui élimine entièrement le rôle de l'observateur ;

- qui n'est pas réfutée par les théorèmes sur les variables cachées comme celui de la Sect.2.2;

- qui rend compte de toutes les expériences justifiant la mécanique quantique ;

- qui permet de comprendre le rôle « actif » de l'appareil de mesure, c'est-àdire l'intuition de Bohr (mais sans en faire un a priori philosophique) ;

- qui explique, dans la mesure où c'est possible, la non-localitée

\section{I Les équations de la théorie de de Broglie-Bohm}

Pour expliquer cette théorie, commençons par l'expérience bien connue des deux trous : on envoie des électrons (un par un en principe) vers un mur dans lequel deux

(6) Nous n'aborderons pas cet aspect de la théorie. Voir [6] chapitre 4 et section 5.2.I pour une discussion de la non-localité. 




(a)

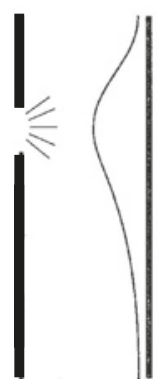

(b)

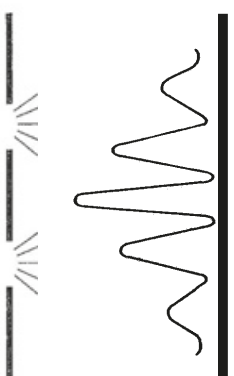

(c)

Figure 5. Expérience des deux trous.

trous peuvent être ouverts ou fermés et l'on détecte les électrons sur un deuxième mur, derrière le premier. Dans la figure 5 , on dessine la densité de détection des électrons sur le deuxième mur selon qu'un trou est ouvert (a), ou l'autre (b) ou les deux (c).

La façon habituelle de décrire cette expérience est de dire que, lorsqu'un seul trou est ouvert, les électrons se comportent comme des particules (parties (a) et (b) de la figure 5), et lorsque les deux trous sont ouverts, ils se comportent comme des ondes (partie (c) de la figure 5). Mais comment les électrons peuvent-ils être à la fois des ondes et des particules? Et comment peuvent-ils savoir à l'avance combien de trous sont ouverts et comment ils doivent se comporter?

On pourrait répondre : élémentaire mon cher Bohr ! Ce sont des particules guidées par des ondes.

Dans la théorie de de Broglie-Bohm, l'état complet d'un système physique est une paire (| état quantique $>, X$ ), où $\mid$ état quantique $>$ est l'état usuel et $X=\left(X_{1}, \ldots, X_{\mathrm{N}}\right)$ représente les positions de particules qui existent indépendamment du fait qu'on les « regarde » ou qu'on les « mesure ».

Ces positions sont les « variables cachées » en ce sens qu'elles ne sont pas inclues dans la description purement quantique | état quantique $>$, mais elles ne sont nullement cachées : ce sont ces positions que l'on détecte directement, par exemple sur l'écran dans l'expérience des deux trous.

L'évolution temporelle de l'état est composée de deux lois :

I. L'évolution usuelle de | état quantique >, pour tous les temps et en toutes circonstances que l'on « mesure » quelque chose ou non. Si l'état est simplement la fonction d'onde | état quantique $>=\Psi\left(x_{1}, \ldots, x_{N}\right)$, on a l'équation de Schrödinger : 
$i \partial_{t} \Psi=\mathcal{H} \Psi$

où

$$
\mathcal{H}=-\frac{1}{2} \Delta+V
$$

est l'hamiltonien quantique ( $\left.\Delta=\sum_{i=1}^{N} \frac{\partial^{2}}{\partial x_{i}^{2}}\right)$ avec, pour simplifier, $\hbar=1$, et toutes les masses égales à I. L'état quantique n'est jamais réduit et ne fait jamais de « saut ».

2. Les positions des particules évoluent au cours du temps $X=X(t)$ selon une équation pilote déterminée par l'état quantique : leur vitesse est une fonction de la fonction d'onde. Si l'on écrit ${ }^{7}$ :

$\Psi\left(x_{1}, \ldots, x_{N}\right)=R\left(x_{1}, \ldots, x_{N}\right) e^{i S\left(x_{1}, \ldots, x_{N}\right)}$,

alors :

$\frac{d X_{k}(t)}{d t}=\nabla_{k} S\left(X_{1}(t), \ldots, X_{N}(t)\right)$.

Ou encore :

$$
\frac{d X_{k}(t)}{d t}=V_{\Psi}^{k}(\mathbf{X}(t))=\frac{\operatorname{Im}\left(\Psi^{*} \nabla_{k} \Psi\right)}{\Psi^{*} \Psi}\left(X_{1}(t), \ldots, X_{N}(t)\right) .
$$

L’origine de cette équation n'a rien de mystérieux ; elle est la forme :

$$
\frac{d \mathbf{X}}{d t}=\frac{\mathbf{J}}{\rho}
$$

où $\mathrm{J}=\operatorname{Im}\left(\Psi^{*} \nabla \Psi\right)$ est le courant associé à la « conservation » de la probabilité $\rho=|\Psi|^{2}$ :

$$
\frac{\partial \rho}{\partial t}+\nabla \cdot \mathbf{J}=0
$$

équation qui se déduit aisément de l'équation de Schrödinger (13).

On peut penser à $\Psi$ comme étant analogue à l'hamiltonien en mécanique classique : celui-ci est aussi défini partout (sur l'espace de phase) et guide le mouvement des particules là où elles se trouvent.

(7) Nous utilisons des lettres minuscules pour désigner les arguments génériques de la fonction d'onde et des lettres majuscules pour les positions effectives des particules. 


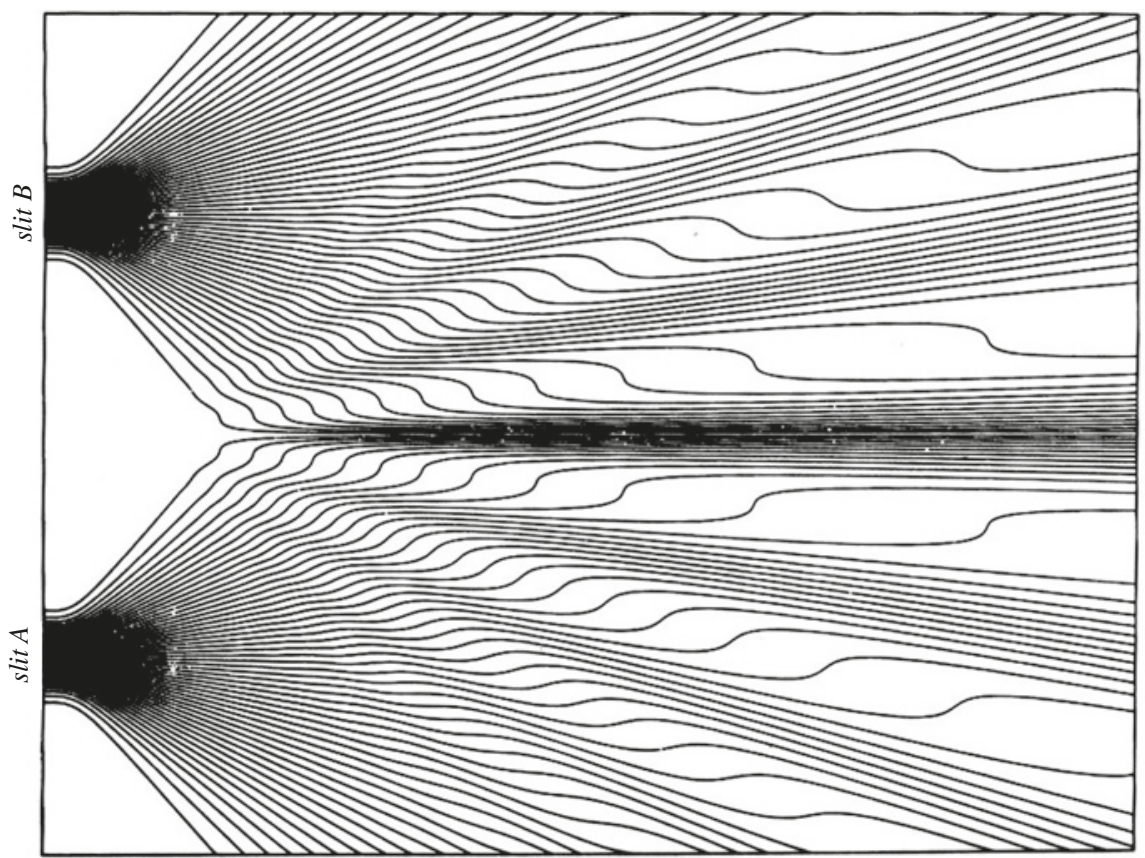

Figure 6. Les trajectoires de la dynamique de de Broglie-Bohm dans l'expérience des deux trous. Calcul numérique dû̀ à [18].

Bohm a réécrit cette équation sous la forme d'une équation du deuxième ordre dans le temps, c'est-à-dire d'une équation de Newton avec un potentiel modifié : $V_{\text {class }} \rightarrow V_{\text {class }}+V_{Q}(\Psi)$ où $V_{Q}(\Psi)=\frac{-\Delta|\Psi|}{2|\Psi|}$. Si l'on tient compte du $V_{Q}(\Psi)$, on peut raisonner plus ou moins « classiquement ».

Revenons à l'expérience des deux trous : il existe des solutions numériques de la dynamique de de Broglie-Bohm dans ce cas-là, voir figure 6 .

On remarquera que le mouvement dans le vide au-delà des trous est hautement non classique! La première loi de Newton (mouvement rectiligne uniforme en l'absence de forces) n'est pas respectée. Si l'on raisonne, comme le faisait Bohm, en terme de potentiels, au-delà des trous $V_{\text {class }}=0$, mais $V_{Q}(\Psi) \neq 0$, vu que $V_{Q}$ dépend de la fonction d'onde, qui se propage au-delà des trous.

Notons que l'on peut déterminer a posteriori le trou par lequel la particule est passée ! Remarquons aussi la présence d'une ligne nodale : comme il existe une symétrie entre le haut et le bas de l'image, le gradient de $\Psi$ est tangent à la ligne séparant ces deux parties de l'image : donc, par (I5), la vitesse de la particule est aussi tangente à cette ligne et les particules ne peuvent pas la traverser. 
Il est intéressant de comparer cette solution numérique à une expérience publiée dans Science en juin 20II [12], et qui montre des trajectoires de photons obtenues à partir d'une série de mesures dite "faibles », qui sont des mesures indirectes. Le profil de ces trajectoires est qualitativement très similaire à celui de la figure 6 .

Dans un manuel de mécanique quantique assez standard, on peut lire :

II est clair que ce résultat [l'expérience à deux trous] ne peut d'aucune manière s'accorder avec la conception du mouvement des électrons sur une trajectoire. [... ] En mécanique quantique, la notion de trajectoire d'une particule n'existe pas.

Landau et Lifshitz [13, p. 8]

Mais, est-ce si clair que cela au vu de ce qui précède ? Bell a bien énoncé le caractère naturel de la théorie de de Broglie-Bohm dans le cadre de l'expérience des deux trous :

N'est-il pas évident, étant donné la petitesse des scintillations sur l'écran, que nous avons affaire à une particule? Et n'est-il pas évident, étant donné les franges d'interférence et de diffraction, que le mouvement de la particule est dirigé par une onde? De Broglie a montré en détail comment le mouvement de la particule, passant par seulement un des trous de l'écran pouvait être influencé par des ondes se propageant à travers les deux trous. Et influencé d'une façon telle que la particule ne va pas là où les ondes s'annulent, mais est attirée là où elles coopèrent. Cette idée me semble si naturelle et simple pour résoudre le dilemme onde-particule, que le fait qu'elle soit si généralement ignorée me paraît être un grand mystère.

John Bell [3, p. 191]

Pour comprendre pourquoi la théorie de de Broglie-Bohm reproduit les prédictions quantiques usuelles, il faut utiliser une conséquence fondamentale de cette dynamique : l'équivariance, qui est illustrée par la figure 7 , où chaque ligne décrit une trajectoire possible.

Si la distribution initiale des trajectoires est donnée par une densité $\rho_{0}$, la densité à un temps ultérieur sera donnée par $\rho_{t}$ :

$$
\rho_{0}(x)=|\Psi(x, 0)|^{2} \rightarrow \rho_{t}(x)=|\Psi(x, t)|^{2},
$$

où $\Psi(x, t)$ provient de l'équation de Schrödinger (I3), et $\rho_{t}(x)$ provient de l'équation pilote $(15)^{8}$. Cela se déduit facilement des équations $(17,18)$ (voir [6] Appendix 5.C pour plus de détails).

(8) Pour tout système dynamique déterministe, où une condition initiale $x_{\mathrm{o}}$ donne lieu à un état $x_{t}$ au temps $t$, on peut définir l'évolution d'une densité de particules par la formule : $\rho_{t}(x)=\rho_{o}\left(x_{o}(x, t)\right)$ où $x_{o}(x, t)$ est la condition initiale au temps 0 qui donne lieu à l'état $x_{t}=x$ au temps $t$. 


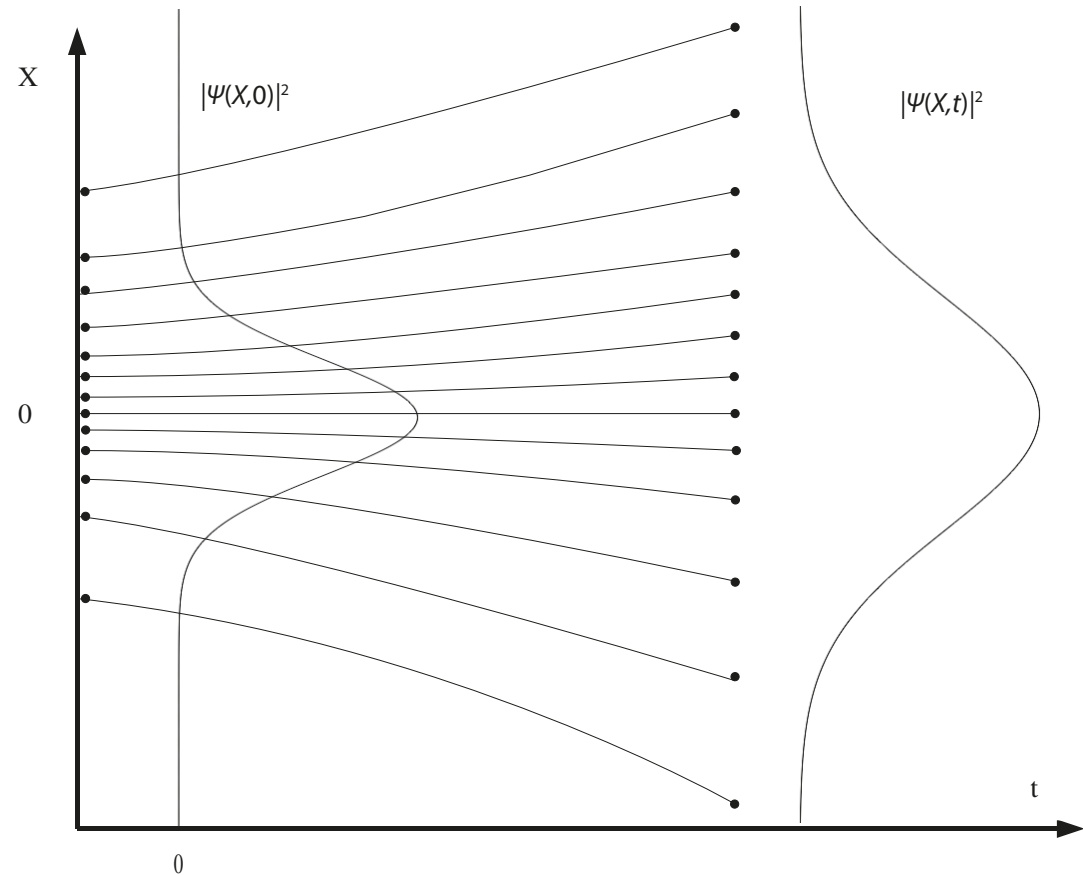

Figure 7. Illustration de l'équivariance de la dynamique de de Broglie-Bohm.

Dans la figure 7 on a représenté une distribution aléatoire de positions initiales dont la densité est approximativement donnée par $|\Psi(x, 0)|^{2}$ (où la variable $x$ est indiquée sur l'axe vertical) et on voit qu'en un temps ultérieur $t$ cette densité sera approximativement donnée par $|\Psi(x, t)|^{2}$, chaque ligne représentant le résultat de la dynamique de de BroglieBohm, pour la position initiale indiquée sur l'axe vertical.

À cause de l'équivariance, les prédictions quantiques pour les mesures de positions seront vérifiées si l'on suppose que la densité des distributions initiales des positions satisfait à $\rho_{0}(x)=|\Psi(x, 0)|^{2}$. Cette hypothèse sur les distributions initiales est appelée l'équilibre quantique ; sa justification est un peu trop longue pour être discutée ici (voir [8] ou [6] section 5.I.7).

Dans la théorie de de Broglie-Bohm, l'état quantique $\Psi$ a donc un double statut :

- il engendre, à travers les équations (I5) ou (I6) le mouvement des particules ;

- il gouverne également la distribution statistique des positions des particules, donnée par $|\Psi|^{2}$. 
On peut comparer l'état quantique avec l'hamiltonien en physique classique, qui engendre le mouvement des particules à travers les équations de Hamilton et donne également les distributions statistiques à l'équilibre. Formellement, cette dernière analogie est la suivante :

$$
\mathcal{H} \sim-\log \Psi \text { et }|\Psi|^{2} \sim \exp (-\beta \mathcal{H}), \text { avec } \beta=2 .
$$

Dans la théorie de de Broglie-Bohm, les seules « variables cachées » qui sont introduites sont les positions des particules. C'est pourquoi la théorie n'est pas réfutée par les théorèmes sur l'impossibilité des variables cachées, qui supposent l'existence d'une fonction $v$ définie sur un ensemble "d'observables », et pas sur une seule comme ici. II nous reste à comprendre comment fonctionnent les mesures d'observables autres que les positions. Nous allons envisager d'abord le spin et ensuite le moment.

\subsection{La « mesure » du spin dans la théorie de de Broglie-Bohm}

Une conséquence des théorèmes sur la non existence de variables cachées vu dans la section 2.2 est que ce qu'on appelle une mesure - autre que les mesures de position - ne mesure (en général) aucune propriété préexistante de la particule. Pour comprendre cela dans la théorie de de Broglie-Bohm, considérons la mesure du spin d'une particule dans un état superposé $\mid 1 \uparrow>$ et $\mid 1 \downarrow>$, que nous décrivons de façon très idéalisée :

$$
\Psi(z)(|1 \uparrow>+| 1 \downarrow>)
$$

$z$ étant la direction verticale (voir la figure 8). Nous supposerons que la partie spatiale de l'état, c'est-à-dire la fonction d'onde $\Psi(z)$ est symétrique : $\Psi(z)=\Psi(-z)$. Ce qui implique que la ligne $z=0$ est une ligne nodale, à travers laquelle le gradient de $\Psi(z)$ est nul et que donc les particules ne peuvent pas franchir, par (I5) (la situation est similaire à celle de la figure 6). $\Psi$ est aussi une fonction de la variable horizontale $x$ (la particule effectue un mouvement vers la droite dans la direction $x$ ), mais nous laissons de côté cette variable.

Dans la figure 8, $H$ désigne un champ magnétique ; les disques représentent (de façon très idéalisée) le support de la partie spatiale de la fonction d'onde. La partie $\mid 1 \uparrow>$ de l'état quantique va toujours dans la direction du champ (ce qui donne lieu à l'état $\Psi(z-t) \mid 1 \uparrow>$ et la partie $\mid 1 \downarrow>$ va toujours dans la direction opposée $\Psi(z+t) \mid 1 \downarrow>$. Mais la particule, si elle est initialement située dans la partie supérieure de la fonction d'onde, va toujours aller vers le haut, à cause de l'existence d'une ligne nodale $z=0$, due à la symétrie de la fonction d'onde, que la particule ne peut pas franchir, comme dans la figure 6.

Donc, si l'on renverse le sens du champ, comme dans la figure 9, la particule va encore aller vers le haut. Mais alors, ce qui était « spin up » devient « spin down », bien que l'on « mesure » la même « observable », c'est-à-dire le spin dans la même direction dans les deux dispositifs, mais avec des arrangements différents de l'appareil. 


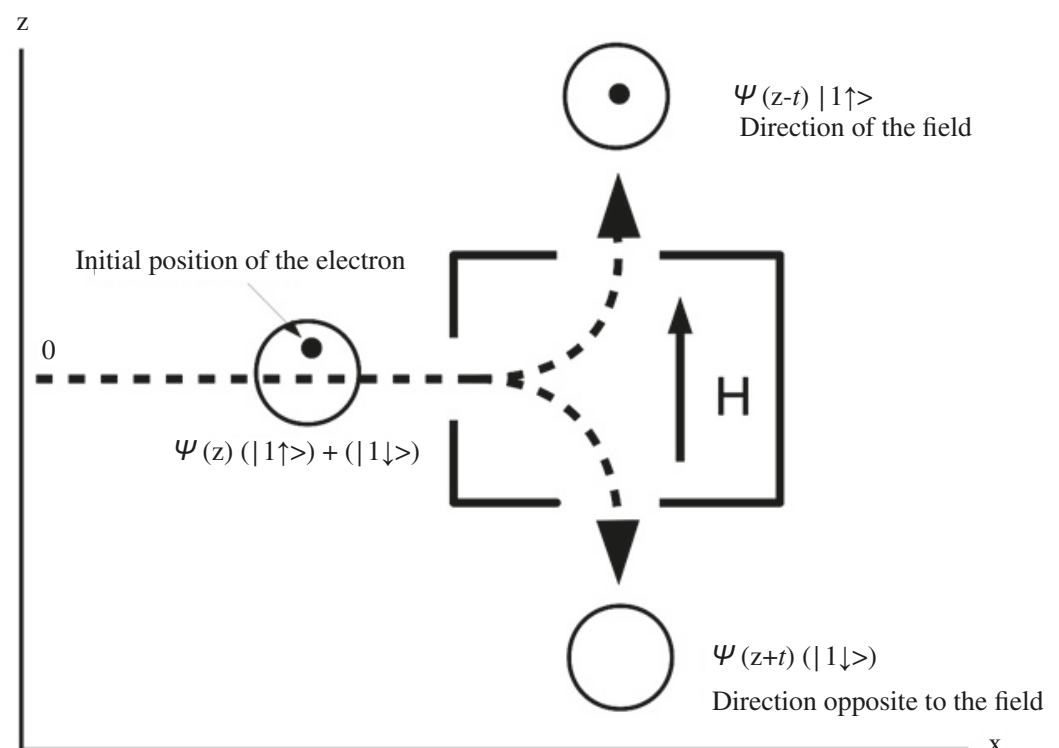

Figure 8. Une mesure de spin idéalisée.

Donc, l'appareil de mesure n'est pas « passif » (il n'enregistre pas simplement quelque chose de préexistant à la mesure) mais « actif ». Ceci justifie l'intuition de Bohr, mais en l'incorporant dans la théorie même, pas comme un deus ex machina.

Notons aussi que les deux parties de la fonction d'onde dans les figures 8 et 9 continuent à évoluer selon les équations habituelles. Mais la particule est guidée uniquement par la partie de la fonction d'onde dans le support de laquelle elle se trouve. C'est-à-dire qu'on peut, en pratique et dans certains cas, réduire la fonction d'onde et ne garder que la partie dans le support de laquelle la particule se trouve. Dans les figures 8 et 9 , il se pourrait que l'on recombine ultérieurement les deux parties de la fonction d'onde et par conséquent on ne peut pas en oublier une (celle dans le support de laquelle la particule ne se trouve pas).

Mais on peut montrer que lorsque la particule interagit avec un appareil macroscopique et que l'on obtient un état du type (6), alors la recombinaison des fonctions d'onde est en pratique impossible ${ }^{9}$ et on peut, de nouveau en pratique, ne garder que la partie de la fonction d'onde dans le support de laquelle elle se trouve si l'on veut analyser son

(9) Comme mentionné dans la Sect.2.I, c'est ce qu'on appelle la décohérence. Il est important de souligner que, si cette notion est essentielle pour comprendre pourquoi ilya en pratique une réduction de l'état quantique dans la théorie de de Broglie-Bohm, elle n'est nullement suffisante à elle seule, puisque rien ne distingue, dans le formalisme usuel, les deuxparties de la fonction d'onde, même si celles-ci « décollèrent». Dans la théorie de de Broglie-Bohm, au contraire, ces deux parties sont différentiées par le fait que la particule est dans le support d'une seule d'entre-elles. 


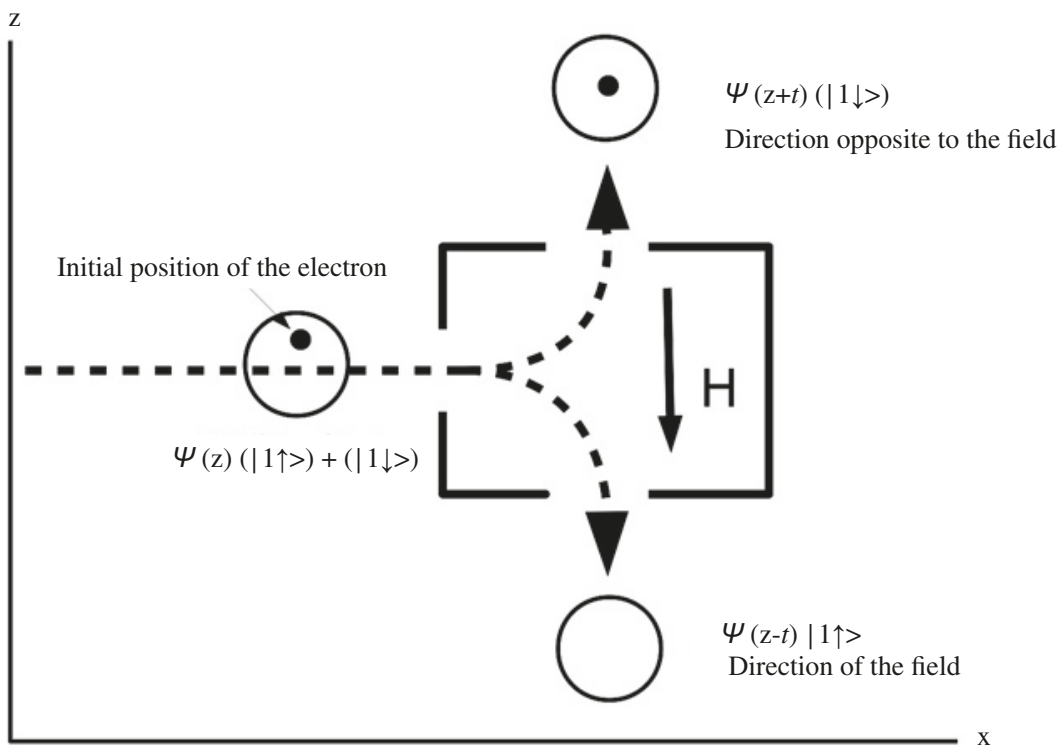

Figure 9. Une mesure de spin idéalisée avec le champ renversé par rapport à la figure 8.

comportement ultérieur. En ce sens, on effectue une « réduction » de l'état quantique et cette opération est « émergente », un peu comme l'irréversibilité des lois macroscopiques en physique classique ${ }^{10}$.

\subsection{La " mesure " du moment dans la théorie de de Broglie-Bohm}

Qu'en est-il de la « mesure du moment » ? Si, dans la théorie de de Broglie-Bohm, les particules ont une trajectoire, elles doivent aussi avoir une vitesse à tout instant. Mais est-ce que la possession simultanée de ces deux propriétés n'est pas en contradiction avec les inégalités de Heisenberg?

Considérons un exemple très simple pour illustrer ce qui se passe, à savoir une particule en une dimension spatiale avec la fonction d'onde initiale $\Psi(x, 0)=\pi^{1 / 4} \exp \left(-x^{2} / 2\right)$. Comme cette fonction est réelle, sa phase $S$ est nulle et toutes particules sont au repos (par l'équation (I5) :

$$
\left.\frac{d}{d t} X(t)=\frac{\partial S(X(t), t)}{\partial x}\right)
$$

(I0) Voir [6] section 5.I.6 pour plus de détails. 
Néanmoins la mesure du moment $p$ doit avoir, d'après les prédictions quantiques usuelles, une distribution de probabilité dont la densité de distribution est donnée par le carré de la transformée de Fourier de $\Psi(x, 0)$, c'est-à-dire par $|\hat{\Psi}(p)|^{2}=\pi^{-1 / 2} \exp \left(-p^{2}\right)$.

N'y a-t-il pas là une contradiction ?

Pour y répondre, il faut d'abord se demander comment on mesure ce moment. Une façon de faire est de laisser évoluer la particule et de détecter sa position asymptotique $X(t)$ quand $t \rightarrow \infty$. On a alors $p=\lim _{t \rightarrow \infty} \frac{X(t)}{t}$ (si l'on pose la masse $m=1$ ).

Considérons l'évolution libre de la fonction d'onde initiale $\Psi(x, 0)=\pi^{1 / 4} \exp \left(-x^{2} / 2\right)$. On a comme solution de l'équation de Schrödinger (13) avec cette condition initiale :

$$
\Psi(x, t)=\frac{1}{(1+i t)^{1 / 2}} \frac{1}{\pi^{1 / 4}} \exp \left[-\frac{x^{2}}{2(1+i t)}\right]
$$

et donc

$$
|\Psi(x, t)|^{2}=\frac{1}{\sqrt{\pi\left[1+t^{2}\right]}} \exp \left[-\frac{x^{2}}{1+t^{2}}\right] .
$$

Si l'on écrit $\Psi(x, t)=R(x, t) \exp [i S(x, t)]$, on a (à une constante en $x$ près) :

$$
S(x, t)=\frac{t x^{2}}{2\left(1+t^{2}\right)},
$$

et l'équation pilote (I5) devient ici :

$$
\frac{d}{d t} X(t)=\frac{t X(t)}{1+t^{2}}
$$

dont la solution est :

$$
X(t)=X(0) \sqrt{1+t^{2}}
$$

Ceci nous donne la dépendance explicite de la position en fonction du temps. Si la particule est initialement en $X(0)=0$, elle ne bouge pas ; sinon elle se déplace asymptotiquement quand $t \rightarrow \infty$, comme $X(t) \sim X(0) t$.

La densité de probabilité que $X(t)=x$ est donnée par $|\Psi(x, t)|^{2}$ (par équivariance). Donc, la probabilité que le résultat de la « mesure » du moment appartienne à $A \subset \mathbb{R}$ est

$$
\lim _{t \rightarrow \infty} \int_{A t}|\Psi(x, t)|^{2} d x
$$


Par un changement de variable $x=p t$, on a :

$$
\int_{A t}|\Psi(x, t)|^{2} d x=t \int_{A}|\Psi(p t, t)|^{2} d p
$$

et, par (22), on obtient :

$$
t \int_{A}|\Psi(p t, t)|^{2} d p=t \int_{A} \frac{1}{\sqrt{\pi\left(1+t^{2}\right)}} \exp \left(-\frac{p^{2} t^{2}}{1+t^{2}}\right) d p
$$

dont la limite, quand $t \rightarrow \infty$ est

$$
\int_{A} \pi^{-1 / 2} \exp \left(-p^{2}\right) d p=\int_{A}|\widehat{\Psi}(p, 0)|^{2} d p
$$

Ce qui est la prédiction quantique! Mais cela ne mesure pas la vitesse initiale (qui est nulle pour toutes les particules).

Donc, les particules ont bien une trajectoire et, à tout moment, une position et une vitesse.

Mais si l'on comprend comment fonctionnent les « mesures », il n'y a aucune contradiction entre la théorie de de Broglie-Bohm et les prédictions quantiques, en particulier avec les inégalités de Heisenberg". Celles-ci sont des relations entre les variances des résultats de mesure, mais n'impliquent rien sur ce qui existe ou n'existe pas en dehors des mesures, puisqu'elles sont des conséquences mathématiques du formalisme quantique et que celui-ci ne dit rien sur le Monde en dehors des mesures.

La leçon à tirer de ce qui précède est que toute mesure autre qu'une mesure de position est une interaction entre le système physique microscopique et l'appareil de mesure macroscopique, mais que ce n'est que dans la théorie de de Broglie-Bohm que l'on peut comprendre comment cette interaction fonctionne.

\section{Conclusions}

Finalement on peut se demander quelle est la relation entre la théorie de de BroglieBohm et la mécanique quantique ordinaire? Une réponse brève serait de dire que c'est la même théorie!

Mais alors, à quoi bon la théorie de de Broglie-Bohm ?

(II) Contrairement à ce que pense, entre autres, Nicolas Gisin [10, p. 75] : « S'il y a des positions cachées, il doit donc y avoir aussi des vitesses cachées. Mais cela contredit le principe d'incertitude d'Heisenberg, principe qui est à la base du formalisme quantique [...] ». 
Plus précisément, l'une (la théorie de de Broglie- Bohm) est une théorie sur le Monde, l'autre (la mécanique quantique ordinaire) non. Elle ne se présente d'ailleurs pas ainsi, mais comme un algorithme permettant de calculer les résultats de mesure!

La mécanique quantique ordinaire est simplement la théorie de de Broglie-Bohm tronquée : on oublie les trajectoires, cela n'affecte en rien les prédictions empiriques (qui sont en fait une conséquence de la théorie de de Broglie-Bohm), et cela crée « simplement » quelques bibliothèques remplies de confusions, de mysticisme et de mauvaise philosophie.

À part cela (clarifier nos concepts), la théorie de de Broglie-Bohm n'a aucune utilitél'

John Bell a expliqué cela en détail :

Pourquoi y a-t-il cette nécessité de faire référence aux 'appareils' quand nous discutons des phénomènes quantiques? Les physiciens qui ont été les premiers à rencontrer ces phénomènes les ont trouvés si bizarres qu'ils ont perdu tout espoir de les décrire en termes de concepts ordinaires comme l'espace et le temps, la position et la vitesse. Les pères fondateurs de la théorie quantique ont même décidé qu'aucun concept ne pourrait être trouvé qui permettrait une description directe du monde quantique. Ainsi, la théorie qu'ils ont établie avait seulement pour but de décrire systématiquement la réponse de l'appareil. Et que faut-il de plus, après tout, dans les applications? [...] Le 'problème' est le suivant: comment le Monde doit-il être divisé exactement entre un appareil parlable ... dont on peut parler ... et un système quantique non parlable, dont on ne peut pas parler? Combien d'électrons, ou d'atomes ou de molécules, faut-il pour constituer un 'appareil' ? Les mathématiques de la théorie ordinaire requièrent une telle division, mais ne disent rien sur la façon de la faire. En pratique, la question est résolue par des recettes pragmatiques qui ont survécu à l'épreuve du temps, appliquées avec discernement et avec un bon goût né de l'expérience. Mais est-ce qu'une théorie fondamentale ne devrait pas permettre une formulation mathématique exacte?

À mon avis, les pères fondateurs avaient tort sur ce point. Les phénomènes quantiques n'excluent pas une description uniforme des mondes micro et macro, ... du système et de l'appareil. II n'est pas essentiel d'introduire une vague division du Monde de ce type.

Cela a été indiqué déjà en 1926 par de Broglie, quand il a répondu à l'énigme onde ou particule?

par onde et particule.

(I2) Ce n'est pas tout à fait exact : il n'y a pas de nouvelles prédictions de la théorie, mais elle a plusieurs applications concrètes, dans les simulations numériques de la fonction d'onde en chimie quantique [22], dans les simulations des problèmes à plusieurs corps [19], ou dans les approximations semi-classiques, (voir [2I] et les références dans cet article). 
Mais, lorsque cela fut complètement clarifié par Bohm en 1952, peu de physiciens théoriciens voulaient en entendre parler. La ligne orthodoxe semblait entièrement justifiée par les succès pratiques. Même aujourd'hui, l'image de de Broglie-Bohm est généralement ignorée, et n'est pas enseignée aux étudiants. Je trouve que c'est une grande perte. Car cette image stimule l'esprit d'une façon très salutaire. L'image de de Broglie-Bohm élimine la nécessité de diviser le Monde d'une certaine façon entre système et appareil.

John Bell [3, 170-17I]

\section{Bell se demandait aussi :}

Pourquoi l'image de l'onde-pilote est-elle ignorée dans les ouvrages de mécanique quantique? Ne devrait-elle pas être enseignée, non pas comme l'unique voie, mais comme un antidote à l'autosatisfaction régnante? Pour montrer que l'imprécision, la subjectivité, et l'indéterminisme, ne nous sont pas imposés de force par des faits expérimentaux, mais par un choix théorique délibéré?

John Bell [3, p. 160]

Pour terminer, citons la réaction d'une ex-étudiante en physique, dont les sentiments sont assez proches des miens lorsque j'étais étudiant :

Ce qui m'a toujours intéressé était de comprendre ce que le Monde est. C'est pourquoi j'ai étudié la physique : si la physique est l'étude de la nature, alors pour comprendre la nature, il faut d'abord étudier la physique. Mais mes espoirs ont été déçus par ce qui est (ou semble être) généralement accepté dans beaucoup de départements de physique dans le monde entier : après la mécanique quantique, il faut abandonner l'idée que la physique nous donne une image de la réalité. Au début, j'ai cru que c'était vrai et j'ai été tellement déçue que j'ai décidé d'abandonner mon rêve 'romantique' [...] Mais, à un moment donné, [...] j'ai réalisé que certaines des choses que j'avais acceptées n'étaient pas si manifestement vraies, et j'ai repris l'espoir que la mécanique quantique n'était pas réellement la 'fin de la physique', au sens où je l'entendais. Par conséquent, j'ai commencé une thèse en physique pour élucider la situation. En faisant mon doctorat sur les fondements de la mécanique quantique, j'ai compris que ce que les physiciens considéraient comme étant une vérité inévitable était au contraire une grossière erreur: la mécanique quantique ne nous force pas à abandonner quoi que ce soit, et sûrement pas la possibilité d'étudier la réalité à travers la physique.

Valia Allori' ${ }^{13}$

Citons également le jugement le plus bref sur « Copenhague » :

Une extravagance philosophique dictée par le désespoir

Erwin Schrödinger ${ }^{14}$

(13) Voir son site web : http://www.niu.edu/vallon/background.html.

(14) Cité par Landé dans [14]. 


\section{Références}

[I] J.S. Bell : On the problem of hidden variables in quantum mechanics, Reviews of Modern Physics 38, 447-452 (1966). Réimprimé comme Chap. I dans [3]

[2] J.S. Bell: Against measurement, Physics World 3, 33-40 (1990). Réimprimé comme Chap. 23 dans [3]

[3] J.S. Bell: Speakable and Unspeakable in Quantum Mechanics. Collected Papers on Quantum Philosophy, 2nd edn, with an introduction by Alain Aspect, Cambridge University Press, Cambridge, 2004; Ist edn 1993

[4] D. Bohm: A suggested interpretation of the quantum theory in terms of "hidden variables", Parts I and 2, Physical Review 89, 166-193 (1952)

[5] N. Bohr: Discussion avec Einstein sur les problèmes épistémologiques de la physique atomique. Chapitre 4 dans : N. Bohr, Physique atomique et connaissance humaine, traduction de l'anglais par Edmond Bauer et Roland Omnès. Edition établie par Catherine Chevalley, Gallimard, Paris, 1991. Original en anglais: Discussion with Einstein on epistemological problems in atomic physics. Dans P.A. Schilpp (ed.): Albert Einstein, Philosopher-Scientist, The Library of Living Philosophers, Evanston, Illinois, 1949, 20I-24I

[6] J. Bricmont, Making Sense of Quantum Mechanics, Springer, Switzerland 2016.

[7] L. de Broglie: La physique quantique restera-I-elle in déterministe?, Gauthier-Villars, Paris, 1953

[8] D. Dürr, S. Goldstein and N. Zanghi: Quantum equilibrium and the origin of absolute uncertainty, Journal of Statistical Physics 67, 843-907 (1992)

[9] A. Einstein : Remarks concerning the essays brought together in this co-operative volume. Dans P.A. Schilpp (ed.): Albert Einstein, Philosopher-Scientist, The Library of Living Philosophers, Evanston, Illinois, 1949, 665-688

[10] N. Gisin: L'impensable hasard. Non-localité, téléportation et autres merveilles quantiques. Préface d'Alain Aspect, Odile Jacob, Paris, 2012

[II] S. Kochen and E. P. Specker: The problem of hidden variables in quantum mechanics, Journal of Mathematics and Mechanics 17, 59-87 (1967)

[12] S. Kocsis, B. Braverman, S. Ravets, M.J. Stevens, R.P. Mirin, L.K. Shalm and A.M. Steinberg: Observing the average trajectories of single photons in a two-slit interferometer, Science 332, $1170-1173(2011)$

[13] L. Landau and E. Lifshitz: Mécanique quantique. Théorie non relativiste, Physique théorique, tome III, éditions MIR, Moscou, 1967 
[14] A. Landé, Quantum fact and fiction, American Journal of Physics, 33, Issue 2, 123- 127 (1965)

[15] J.L. Lebowitz: Microscopic origins of irreversible macroscopic behavior. Round Table on Irreversibility at STATPHYS20, Paris, July 22, 1998, Physica A 263, 516-527 (1999)

[16] D. Mermin: Hidden variables and the two theorems of John Bell, Reviews of Modern Physics 65, 803-815 (1993)

[17] A. Peres: Two simple proofs of the Kochen-Specker theorem, Journal of Physics A: Math. Gen. 24, LI75-LI78 (199I)

[18] C. Philippidis, C. Dewdney, B.J. Hiley: Quantum interference and the quantum potential, II Nuovo Cimento B 52, 15-28 (1979)

[19] X.O. Pladevall, J. Mompart (eds): Applied Bohrnian Mechanics: From Nanoscale Systems to Cosmology, Pan Stanford Publishing, Singapore, 2012

[20] E. Schrödinger: Die gegenwärtige Situation in der Quantenmechanik, Naturwis- senschaften 23, 807-812; 823-828; 844-849 (1935). Traduction anglaise : The present situation in quantum mechanics, translated by J.- D. Trimmer, Proceedings of the American Philosophical Society 124, 323-338 (1984). Réimprimé dans: J.A. Wheeler and W.H. Zurek (eds), Quantum Theory and Measurement, Princeton University Press, Princeton, 1983, 152-167

[21] W. Struyve: Semi-classical approximations based on Bohmian mechanics, preprint arXiv: |507.0477| (2015)

[22] R.E. Wyatt, Quantum Dynamics with Trajectories, Springer, New York, 2005 
